\title{
Radiographs for publication
}

\author{
Basil Strickland ${ }^{1}$
}

Although clinical cardiologists make full use of the advances in diagnostic radiology in their daily professional lives, they seldom give sufficient care to the preparation of radiographic illustrations in articles submitted to the Journal. An author should take counsel with his radiologist so that the best original radiograph may be chosen. He should also consult with the medical photographer who is preparing the prints, appraising him of the particular features to be reproduced in each photograph.

Careful 'editing' of the photograph is important so that the object of direct clinical interest is portrayed together with sufficient surrounding 'field' to allow easy and rapid orientation. Photographs of the entire thorax are indicated only where an anatomical landmark or comparison is germane to the clinical discussion. Coned views are often most valuable especially in the demonstration of areas of calcific density or increased transradiency. The vascular pattern of the lungs is especially difficult to reproduce in print, and here a contact print of a relevant portion of the peripheral lung field is usually the only satisfactory method of demonstrating the minutiae of the vascular markings.

\footnotetext{
1 Address: Brompton Hospital, Fulham Road, London SW 36 HP.
}

Overexposure and an insufficient range of contrast values are the commonest major faults in photographs submitted to the Editor. The 'soot and whitewash' print is unacceptable and a much finer technique in processing with more grey shades is required. Considerable losses of image quality are inherent in the process of converting a radiographic image to a photographic print, and great care is called for throughout the entire photographic process from the choosing of the radiograph to the fashioning of the block by the printer.

Radiographic reproductions should be submitted as unmounted, negative ('facsimile') glossy prints. The optimum size for reproduction is "wholeplate', $216 \times 152 \mathrm{~mm}\left(8 \frac{1}{2} \times 6 \frac{1}{2}\right.$ inches $)$ and no print smaller than 'half-plate' will be accepted. Each print should be numbered on the reverse, marked 'top' at the appropriate edge, and the name(s) of the authors added. A soft pen or pencil (e.g. a felt-tip) should be used. Never use a ball-point pen as this indents the print and the marks will show on the block. If attention is to be drawn to particular features, bold arrows should be inserted directly on to the print preferably by Letraset. Where additional clarification is necessary a line drawing tracing of the relevant area may be provided. 